\title{
Analysis of Dual-Band Direction of Arrival Estimation in Multipath Scenarios
}

\author{
Alessandro Cidronali (D), Edoardo Ciervo*(D), Giovanni Collodi $\mathbb{D}^{\text {, }}$, Stefano Maddio (D), Marco Passafiume \\ and Giuseppe Pelosi (D)
}

Citation: Cidronali, A.; Ciervo, E.; Collodi, G.; Maddio, S.; Passafiume, M.; Pelosi, G. Analysis of Dual-Band Direction of Arrival Estimation in Multipath Scenarios. Electronics 2021, 10, 1236. https://doi.org/10.3390/ electronics10111236

Academic Editor: Toshifumi Moriyama

Received: 30 March 2021

Accepted: 20 May 2021

Published: 22 May 2021

Publisher's Note: MDPI stays neutral with regard to jurisdictional claims in published maps and institutional affiliations.

Copyright: (c) 2020 by the authors. Licensee MDPI, Basel, Switzerland. This article is an open access article distributed under the terms and conditions of the Creative Commons Attribution (CC BY) license (https:// creativecommons.org/licenses/by/ $4.0 /)$.

\author{
Department of Information Engineering, University of Florence, Via Santa Marta, 3, 50139 Firenze, Italy; \\ alessandro.cidronali@unifi.it (A.C.); giovanni.collodi@unifi.it (G.C.); stefano.maddio@unifi.it (S.M.); \\ marco.passafiume@unifi.it (M.P.); giuseppe.pelosi@unifi.it (G.P.) \\ * Correspondence: edoardo.ciervo@unifi.it
}

\begin{abstract}
The present paper analyzes the performance of localization systems, based on dual-band Direction of Arrival (DoA) approach, in multi-path affected scenarios. The implemented DoA estimation, which belongs to the so-called Space and Frequency Division Multiple Access (SFDMA) technique, takes advantage of the use of two uncorrelated communication carrier frequencies, as already demonstrated by the authors. Starting from these results, this paper provides, first, the methodology followed to describe the localization system in the proposed simulation environment, and, as a second step, describes how multi-path effects may be taken into account through a set of full-wave simulations. The latter follows an approach based on the two-ray model. The validation of the proposed approach is demonstrated by simulations over a wide range of virtual scenarios. The analysis of the results highlights the ability of the proposed approach to describe multi-path effects and confirms enhancements in DoA estimation as experimentally evaluated by the same authors. To further assess the performance of the aforementioned simulation environment, a comparison between simulated and measured results was carried out, confirming the capability to predict DoA performance.
\end{abstract}

Keywords: indoor localization; directional of arrival; multipath; dual band; full-wave simulation; unmanned ground vehicle; indoor autonomous driving

\section{Introduction}

Indoor localization represents an enabling technology for a wide range of applications where it is necessary to contextualize the position of people, objects and/or vehicles such as Unmanned Ground Vehicles (UGV), in real complex indoor environments. With special reference to logistic scenarios including UGVs, the interaction between the latter and the indoor positioning system may be related to supply UGV requirements in terms of operating environment awareness or support for high-precision autonomous driving [1].

Moreover, it is a matter of fact that Internet of Things (IoT) paradigm can benefit from localization in many applications [2]. Indoor localization will be a key focus also in 5th generation mobile (5G) [3], which will take advantage of the inherently use of massive Multiple Input Multiple Output (MIMO) technology.

In recent years, several studies have been carried out on localization techniques and methodologies, with the common aim of achieving improvements in positional accuracy as well as infrastructure simplifications. It is well known that these objectives are extremely difficult to achieve at the same time. Nevertheless, recent works have proposed solutions to overcome this issue. Specifically, some approaches compensate the lower complexity of the implemented infrastructure with solutions characterized by a higher computational cost enabling accuracy enhancements such as in [4], where likelihood functions are implemented in machine learning (ML) algorithms. Localization methods based on ML are discussed also in [5-7], where in the latter such algorithm is applied to a WiFi dual-band-based 
position system. In this context, processing solutions that can extract the location estimate in the case of limited data availability have also been investigated, as described in [8]. In such approach the localization is computed according to statistical methods based on Gibbs sampling of a mobility model. On the contrary, in $[9,10]$, the authors discuss the use of Switched Beam Antennas to achieve lower infrastructural complexity and more compact nodes.

There is no doubt that all these approaches have contributed to a progressive improvement in terms of localization accuracy. However, in many real-world application scenarios, the presence of very severe multi-path phenomena may limit these enhancements. To mitigate these effects, specific multi-path-oriented techniques have been proposed. Some of these methods involve multi-path modeling in the localization algorithm with the disadvantage of increased computational overhead. In this regard, some ML techniques including multi-path models are described in [11,12], while [13] proposes a localization system based on Angle of Arrival technique assisted by an algorithm used to elaborate multi-path information relying on the image method. With the aim of preferring a simplified infrastructure, also [14] discusses about the exploitation of multi-path signals estimated using JADE algorithm to improve the positioning accuracy using a single receiver. Conversely, other methods prefer to implement a more robust hardware infrastructure against multi-path phenomena, but with the disadvantage of a more complex positioning system. In such context, ref [15] describes a multi-path mitigation method using MIMO beamforming scheme.

Mitigation of adverse effects caused by multi-path is a very challenging problem in all the localization techniques. All the papers cited in this section suggest approaches to attenuate the problem related to this important issue affecting the application in real scenarios. In the present paper, the authors propose an analysis method aimed at fully exploiting the capability of dual-band DoA approach as suggested by [16].

\section{Dual-Band DoA Approach}

DoA is a well-known technique commonly used for localization $[17,18]$ in many communication systems [19] such as WiFi [20], Bluetooth [21] and Ultra-Wide Band [22].

Among all the possible approaches proposed to simplify the positioning infrastructure and improve accuracy, the DoA technique shows the advantage of combining the use of a limited number of nodes with a simple estimation algorithm.

In particular, DoA approaches implementing the Space Division Multiple Access (SDMA) technique, based on phaseless Received Signal Strength Indicator (RSSI), represent efficient and effective solutions for indoor localization applications [23,24]. The integration of RSSI measurement circuits in almost every legacy transceiver $[25,26]$ makes this approach really worthwhile.

Among the various DoA estimation strategies available in the literature, the most effective are those based on Maximum-Likelihood (MLH) criteria [27,28]. These wellknown methods solve the estimation problem by comparing the power of the impinging signal vector with the predicted power distribution, estimated from the beam-set of the anchor antenna [29]. The calculated DoA becomes the one that minimizes a cost function based on the difference between the actual signal and the predicted one. This approach allows real-time estimations, even with ultra-low hardware resources, given the extremely reduced computational cost of the algorithm.

Unfortunately, DoA approaches are heavily influenced by critical multi-path effects [30], since the angular distribution of signals may be widely different from that expected because of undesired signal contributions originating from the latter.

Over the years, solutions to minimize these effects have been proposed and demonstrated. In particular, in previous papers [16,31,32], the authors have suggested a solution based on combination of Frequency Division Multiple Access (FDMA) approach along with RSSI-based DoA techniques. The results illustrated in those papers show an improvement in system performance with respect of complex real-life scenario, hardly affected by 
multi-path issues. Specifically, in [16] the authors shown, through a series of experiments, how a DoA approach based on two uncorrelated frequencies leads to a robust improvement of the DoA estimation.

The idea behind the suggested dual-band DoA solution refers to the assumption that for any given scenario, it is well known that there is a very low probability for signals at two uncorrelated frequencies to be equally affected by multi-path. Unfortunately, the severity of these non-idealities cannot be determined a-priori. It is a matter of fact that it is difficult to fully demonstrate the real benefits of a dual-band approach making use exclusively of measurement-based experiments.

The aim of this paper is to illustrate an analysis environment capable of correctly predicting the improvement introduced by the use of a dual-band system approach in a multi-path affected complex scenario, where the first line of multi-path is the one which downgrade the system performance harder than the other components. Consequently, the core of the proposed environment consists of modeling the first line effects by means of a full-wave simulation. Multipath effects are described making use of a two-ray reflection model exploited in [33]. The introduction of the present technique of analysis gives the system designer an effective a-priori method that reduces the on-site verification step. The use of rigorously modeled full-wave analysis within the simulation environment leads to an excellent agreement, in terms of performance improvements compared with the experimental results already shown in previous literature [16].

\section{Localization System}

This section describes the reference indoor localization system as well as the anchor device modeling. Afterwards, we introduce the dual-band DoA estimation approach that is implemented in the proposed analysis environment based on the one presented in [16].

\subsection{Reference System}

The reference localization system modeled in the present paper is based on the same anchor node illustrated in [16]. A complete block diagram of the system is depicted in Figure 1.

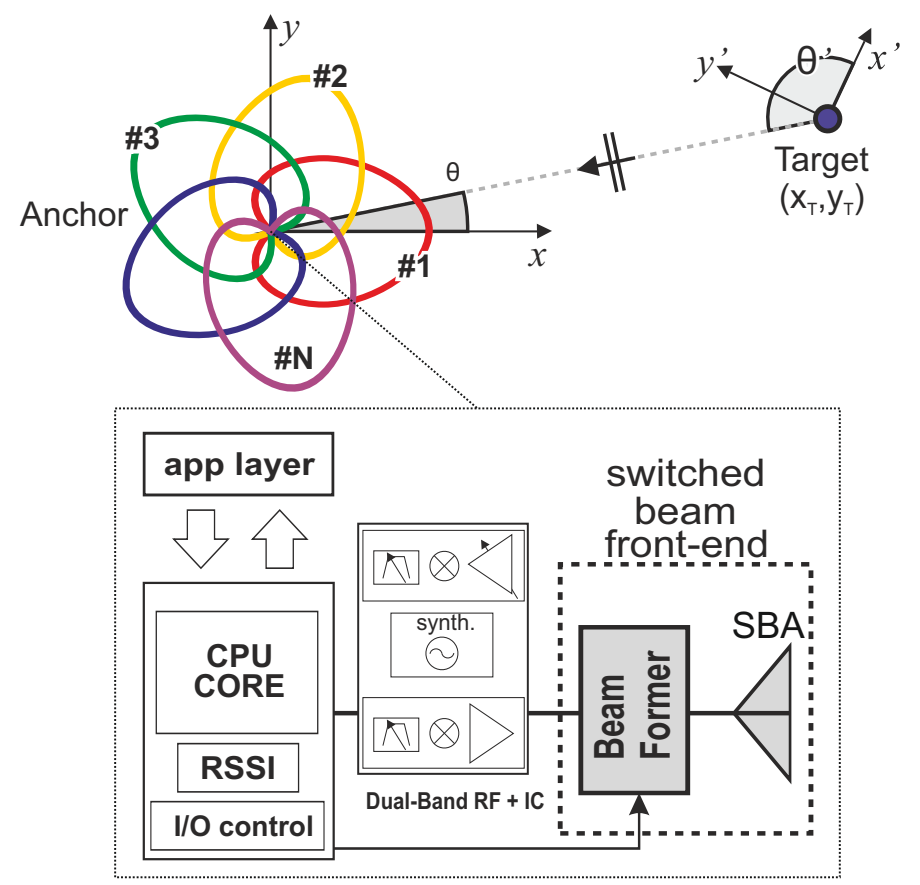

Figure 1. Architecture of the proposed anchor device, based on the one defined in [16] and overview of the localization system. 
The anchor device is based on a radio-frequency transceiver, compatible with the IEEE 802.11x radio technology, and a Switched Beam Antenna (SBA). This configuration is an evolution of the current hardware described in [31].

The node is capable of operating at two different bands, implementing both a dualband wireless transceiver and a dual-band Switched Beam Antennas (SBA). It also integrates a micro-controller which implements the necessary communication and protocol layers. The SBA exploits the combined SFDMA diversity via dual-band antennas operating at $2.45 \mathrm{GHz}$ and $5.2 \mathrm{GHz}$, hence compatible with IEEE 802.11ac radio access technologies. The SBA comprises of eight printed patch antennas, arranged as an octagonal prism as in Figure 2.

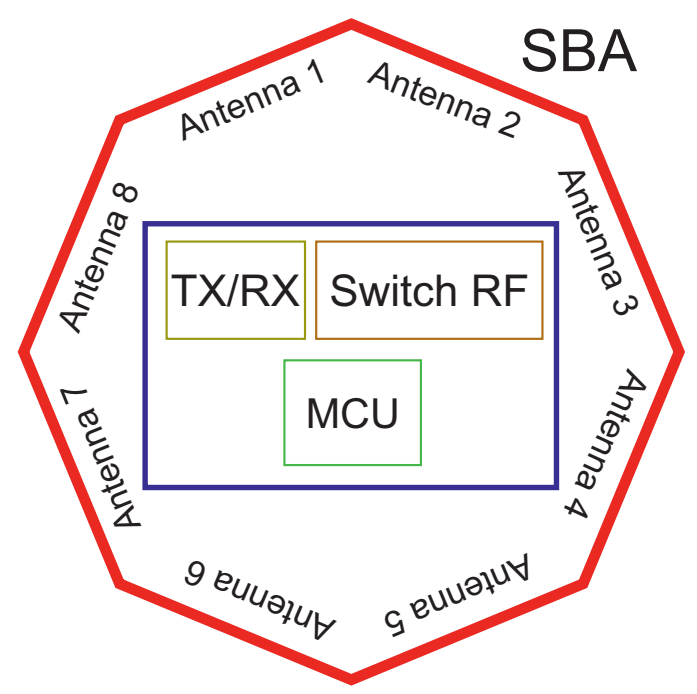

Figure 2. SBA of the proposed anchor device.

This shape is suitable for optimal signal distribution in the plane, thus enabling a precise 2-Dimensional DoA estimation. Elementary antennas operate in circular polarization $(\mathrm{CP})$, permitting robust communication regardless of the relative orientation of the target antenna and effectively reducing multipath interference, as shown in [34-36]. Refer to [37] for a more in-depth analysis of the patch element design.

\subsection{Anchor Device Modeling}

The anchor described in the previous subsection and used in [16] has been modeled in the proposed analysis method.

The radiation patterns of the eight patch antennas implemented in the SBA are considered identical and modeled by means of a cardioid. The latter is obtained via a best-fitting procedure carried on the average value calculated starting from the eight effective antennas radiation patterns [16]. The results for both dual frequencies are shown in Figure 3. Considering dual-band operation, the $\mathrm{n}$-th beam pattern pointing toward a generic direction $\left(\theta_{n}\right)$ at the $i$-th frequency can be written as:

$$
G_{n}^{(i)}(\theta)=G_{n}^{(i)}\left[\frac{1}{2}\left(1+\cos \left(\theta-\theta_{n}\right)\right)\right]^{p_{n}^{(i)}}
$$

The exponents $p_{n}^{(i)}$ control the beamwidth for the $i$-th frequency, while the coefficients $G_{n}^{(i)}$ are the maximum gains. For fixed frequencies, these are nominally identical. However, fabrication tolerance, mutual coupling, channel imbalance, and mechanical arrangement tolerance can affect the beam-set uniformity.

The simulation environment also demands the modeling of all the other functional components that constitute the anchor node. A descriptive approach based on the idealization of their features results sufficiently suitable to the aims of the analysis that we want to 
carry out. On the contrary, the description of the SBA is a critical point since the latter is the core of the system.

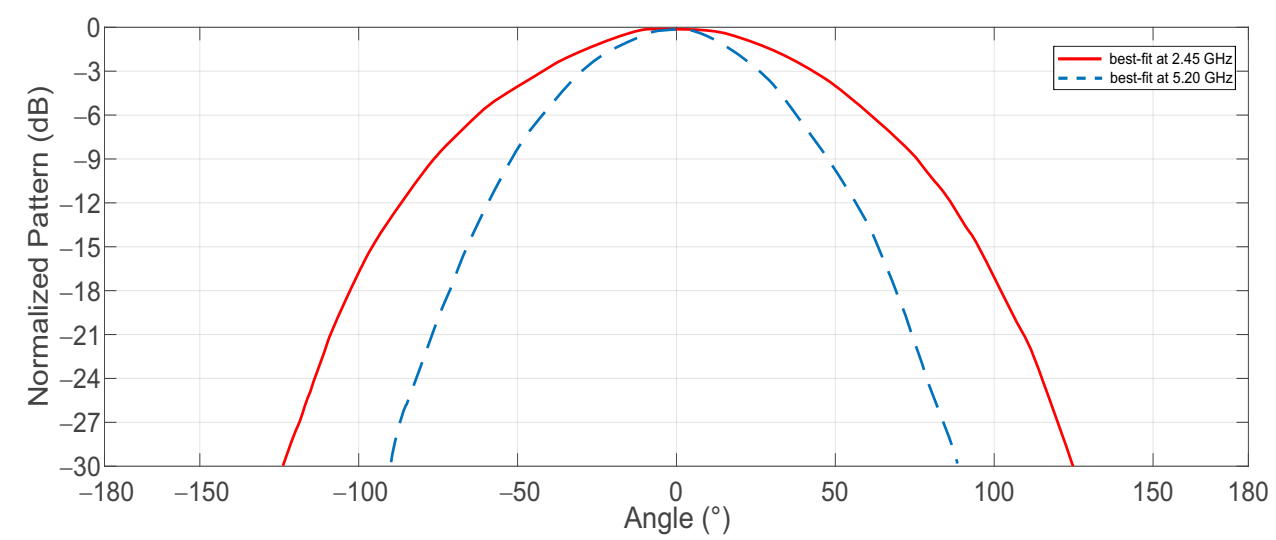

Figure 3. Radiation patterns of the SBA antennas implemented in the simulation environment.

\subsection{DoA Estimation Method}

The azimuthal DoA estimation method implemented in the proposed simulation environment is based on the procedure described in [16,38]. In both papers, this estimation is obtained as a result of a Maximum-Likelihood (MLH) criterion driven by comparing the actual real-time RSSI measurement with the expected signal magnitude, where the latter one is derived from the antenna beam-set.

To better clarify all the issues involved in the DoA estimation method, the following paragraph briefly summarize the key elements of the approach implemented in the present paper. In accordance with Figure 1 , the target, located in the position $\left(x_{T}, y_{T}\right)$, acts as a transmitter of message sequences. The power of the packets sequentially received from the anchor throughout the beam-set, are stored as a vector of RSSI readings. Since the target position is unknown, the angle of view $\theta$ relative to the anchor node is unknown as well. The RSSI signal through the $n$-th beam at the $i$-th frequency of operation, labelled as $S_{n}^{(i)}$, is the result of the following observation model:

$$
S_{n}^{(i)}=G_{n}^{(i)}(\theta)+P_{i n c}^{(i)}\left(D, \theta^{\prime}\right)+n_{n}^{(i)}
$$

where fixed the $i$-th frequency, $G_{n}^{(i)}(\theta)$ is the $n$-th gain pattern through the nominal direction $(\theta)$ of the target with respect to the anchor node reference (Figure 1$)$, and $P_{i n c}^{(i)}\left(D, \theta^{\prime}\right)$ is the power of the wavefront impinging at the anchor section from the distance $D$, through direction described by the $\theta^{\prime}$ angle with respect to the target system reference (Figure 1). Finally, $n_{n}^{(i)}$ is a noise term, which also include all those multi-path effects that usually occur but cannot be explicitly stated mathematically.

With reference to (2) the above-mentioned incident power can be expressed as:

$$
P_{i n c}^{(i)}(D, \theta)=P_{T}^{(i)}+\alpha_{(i)} 10 \log \left(\frac{4 \pi D}{\lambda_{(i)}}\right)+G_{T}^{(i)}\left(\theta^{\prime}\right)
$$

where the additive term $P_{T}^{(i)}$ is the power transmitted by the target node, $\alpha_{(i)}$ is the pathloss exponent, and $G_{T}^{(i)}\left(\theta^{\prime}\right)$ is the gain of the target antenna, defined in its own reference.

In principle, the mathematical solution of the DoA estimation problem may be obtained reverting the relationship in (2), which in turn is a non-linear system in $\theta$. However, being unknown the noise term introduced in the model, the latter cannot be solved in closed form. 
This problem can be easily solved by means of the aforementioned MLH criteria which makes use of the residual term $\mathbf{R}$. As already introduced in [38], at the $i$-th frequency this one is defined as: where $\mathbf{R}^{(i)}$ and $\mathbf{S}^{(i)}$ are the vector form of the gain and the signal in (2).

One of the most popular MLH approaches is the Least Square Estimator (LSE). At the $i$-th frequency, the cost of LSE is defined as:

$$
\mathrm{C}_{\mathrm{LSE}}^{(\mathrm{i})}(\theta)=\left\|\mathbf{R}^{(i)}(\theta)\right\|=\sqrt{\sum_{n=1}^{N}\left(S_{n}^{(i)}-G_{n}^{(i)}(\theta)\right)^{2}}
$$

thus, the estimated azimuthal DoA $\widehat{\theta}$ is identified as the one which satisfies:

$$
\widehat{\theta}^{(i)}=\underset{\theta}{\operatorname{argmin}} \mathrm{C}_{\mathrm{LSE}}^{(\mathrm{i})}(\theta)
$$

As an evolution of the former, in [39] was introduced another more suited estimator for dual-band operation,-based on the variance of the residual. For this approach, called minVAR, the cost function is defined as:

$$
\begin{aligned}
& \mathrm{C}_{\mathrm{MV}}^{(\mathrm{i})}(\theta)=\operatorname{var}\left[\mathbf{R}^{(\mathrm{i})}(\theta)\right]=\operatorname{var}\left[\mathbf{S}^{(i)}-\mathbf{G}^{(i)}(\theta)\right]= \\
& =\frac{1}{N} \sum_{n=1}^{N}\left(\left(S_{n}^{(i)}-G_{n}^{(i)}(\theta)\right)-\frac{1}{N} \sum_{n=1}^{N}\left(S_{n}^{(i)}-G_{n}^{(i)}(\theta)\right)\right)^{2}
\end{aligned}
$$

Same as the previous case, the formal estimation is found with an operator similar to (5), but applied to the cost function (6).

Considering the dual-band operation, the most effective exploitation of the available data is to combine the RSSIs at the two frequencies as a single input vector for the minVAR algorithm. Therefore, the extend gain vector $\mathrm{G}$ becomes:

$$
\mathbf{S}=\left[\mathbf{S}^{1}, \mathbf{S}^{2}\right], \quad \mathbf{G}=\left[\mathbf{G}^{1}, \mathbf{G}^{2}\right]
$$

Operating with the combined data, the actual DoA still emerges as the minimum variance angle, in the sense described in (7).

\section{Simulation Environment: Implemented Scenario}

As previously mentioned in Section 2, the aim of this paper is to propose a simulation environment capable of correctly predicting the improvement due to the use of the aforementioned dual-band system approach. This simulation tool permits the full investigation of the effects of multi-path contributes and their impacts on the estimation results based on a simple but effective model. The basic idea is to recreate a real-life scenario making use of trustful full-wave simulations, thought to be compatible with experimental results presented in [16].

As a first step, the link between the actual anchor $(A)$ and target $(T)$ is simulated by means of Ansys HFSS. The 8-beam SBA and the target antenna are modeled and simulated to match those presented in [31]. The spatial configuration of the whole system is chosen in the same way as that illustrated in the aforementioned manuscript.

In Figure 4 is depicted a representation of multi-path phenomena based on a two-ray reflection model. Starting from this key point, the simulation environment introduces the multipath contributes by means of a second radiating element which acts as an Image (I) antenna and removing the ground plane. This approach is depicted in Figure 5 where the Image antenna is explicitly highlighted and operates as a coherent communication interferer. The insertion of this element allows the accomplishment of a controlled multipath scenario. Consequently, the full-wave simulation requires the use of a total of $8+2=10$ antennas. The resulting $10 \times 10$ scattering matrix describes the coupling between the involved antennas with full-wave accuracy. 


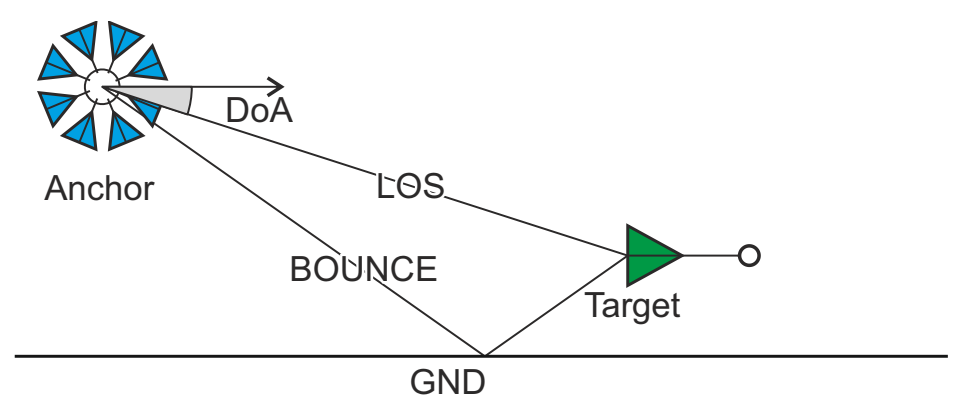

Figure 4. Pictorial rendition of the multipath-impaired scenario for the DoA estimation.

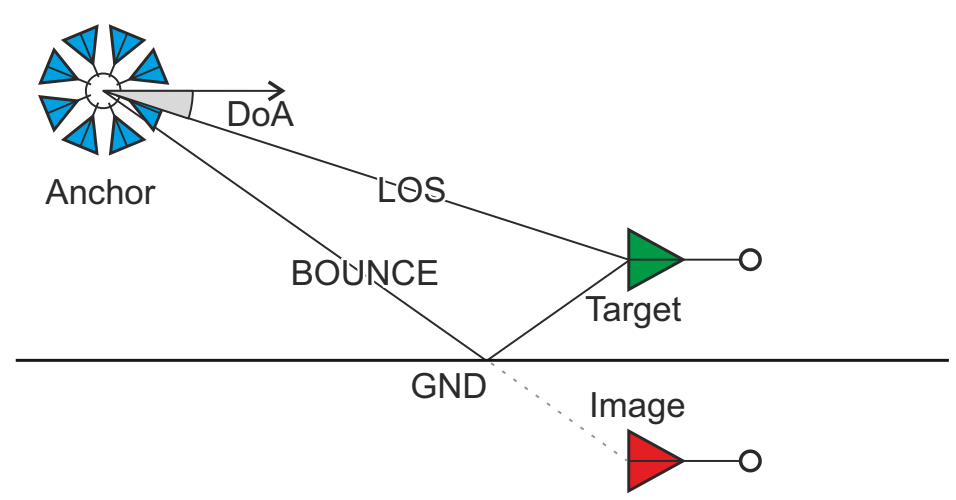

Figure 5. Pictorial rendition of multi-path propagation using an image-source model.

The goal of describing a truly realistic multi-path environment is attained running a series of full-wave simulation. In each of them, the position of $T$ is varied to cover a different scenario. Additionally, the location of $I$ is varied accordingly, to emulate a two-way bounce in a consistent way following an approach that is perfectly coherent with the equivalence theorem.

For each position of $T$ (and therefore $I$ ) the anchor is rotated over $360^{\circ}$ with steps of $5^{\circ}$, meaning 73 full-wave simulations for each relative position. This is coherent with the measurements in [16], which can therefore be used for validation.

Figure 6 shows the proposed controlled multi-path scenario, based on the imagesource model. The EM simulation sets representing the link data may be manipulated interconnecting a set of $7310 \times 10$ scattering matrices with an ideal circuital network composed by a Wilkinson Power Divider (PD), a Phase-Shifter (PS) and an Attenuator (ATT). The resulting circuital network is configured in such a way that the source signal, i.e., the broadcast message, is split between the two branches of the PD connected, respectively, to the Target and the Image antenna. The latter branch enabling the manipulation the of incoming signal by means of a PS and an ATT to create a controllable interference, both in absolute value and phase. As a result, the link between the anchor and target is described by means of a set of $739 \times 9$ scattering matrices, which is in turn manipulated for the setting of ATT and PS using command lines. Thanks to this, the interferer signal may cover a wide range of possible scenario.

In accordance with Figure 6, the attenuation factor on the image antenna branch is indicated with the letter $\chi$, while the relative phase between target and image signal is indicated with $\alpha$.

Referring to Equation (2), the resulting RSSI used in the DoA estimation algorithm include an unavoidable contribution of noise. Formally, the latter consists of two main components. The first one takes into account both thermal noise as well as process errors. The second one is deterministic, and depends on the multi-path direction $\theta_{M P}$, and how the gain terms $G_{n}^{(i)}(\theta)$ and $G_{T}^{(i)}\left(\theta^{\prime}\right)$, as well as the pathloss $L^{(i)}(D)$ including $\alpha_{(i)}$, impact on the signal recombination at the anchor reference, also in dependence of $\chi$ and $\alpha$. The 
proposed controlled multi-path scenario is thought to predict that deterministic part which is usually unknown by the DoA estimation algorithm.

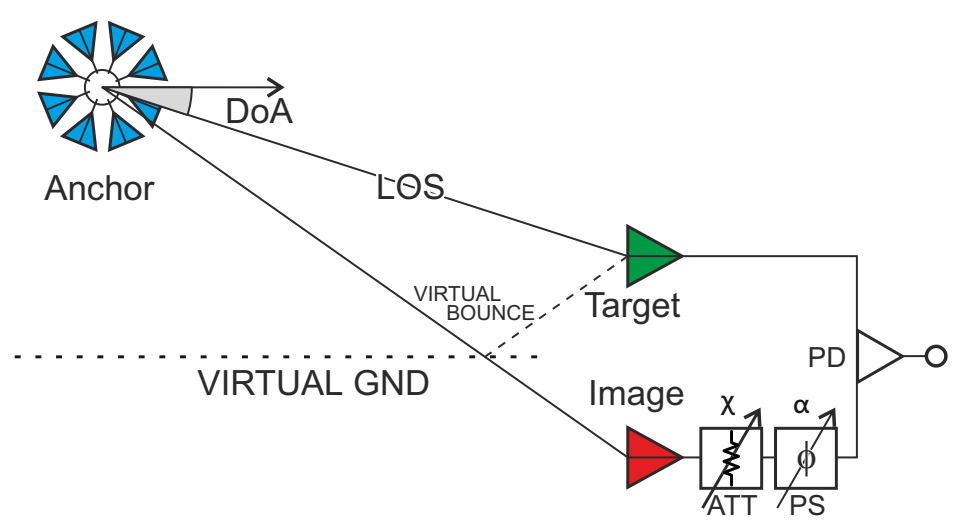

Figure 6. Pictorial rendition of the proposed controlled multi-path scenario.

It is worthwhile to mention that in a real implementation the estimation of RSSI is based on signal-matching strategies, which mitigates the impact of multi-path on actual input data by at least partially filtering out the echo signal. Consequently, the proposed investigation is more severe than a real application in this sense.

With reference to Figure 7 , the $P_{R}^{(i)}$ for $f_{1}=2.45 \mathrm{GHz}$ and $f_{2}=5.2 \mathrm{GHz}$ are reported in the form of a contour plot, in both angle and distance.
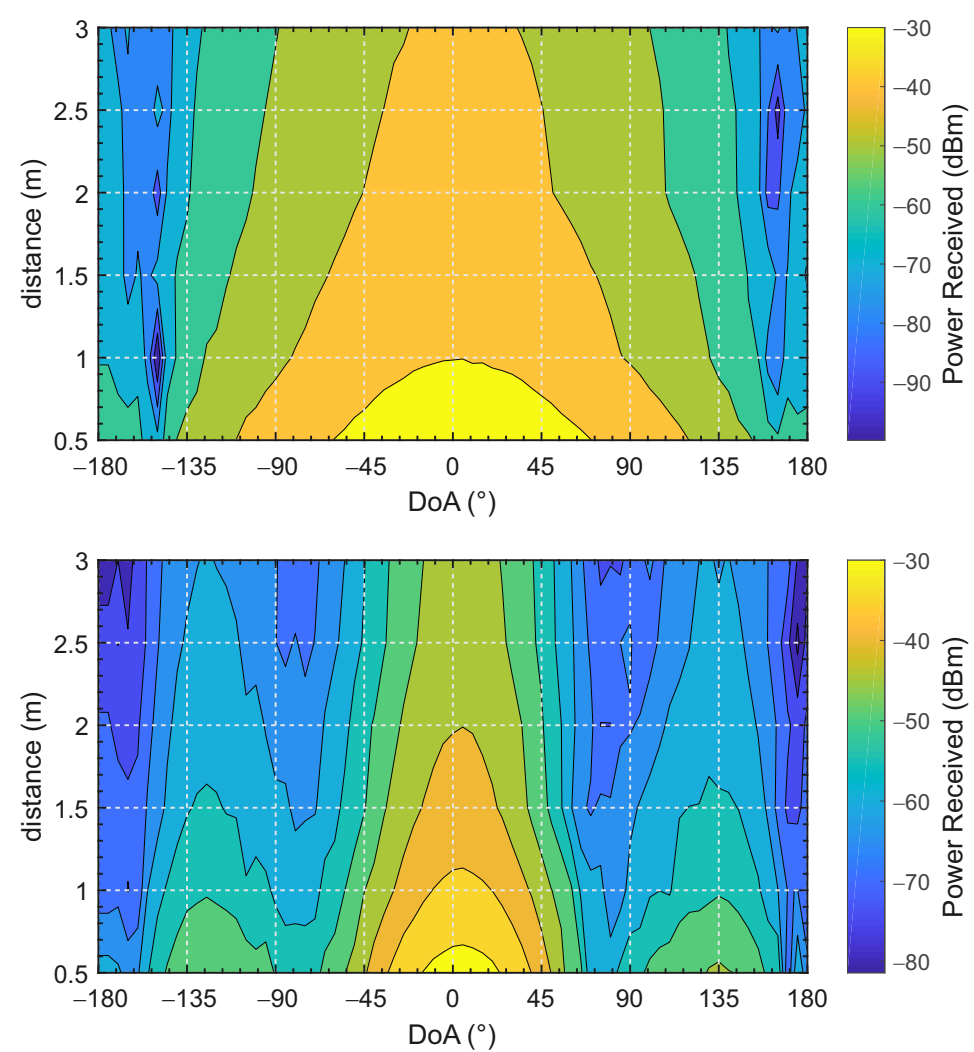

Figure 7. Power distribution relative to antenna \#1 in the unperturbed case, at $2.45 \mathrm{GHz}$ (top) and $5.2 \mathrm{GHz}$ (bottom).

This figure depicts the case when the multi-path signal power $\chi$ is equal to $20 \mathrm{~dB}$, hence the reception is almost identical to the unperturbed case.

Figure 8 shows the same information, but with a very strong image target $(\chi=1 \mathrm{~dB})$. It is easy to notice that the multi-path signal affects deeply the power distribution. Further- 
more, it is interesting that the positions of the nulls is almost complementary in the two cases, so that when one frequency is strongly impaired by the disturbance, the other one is almost unscathed.
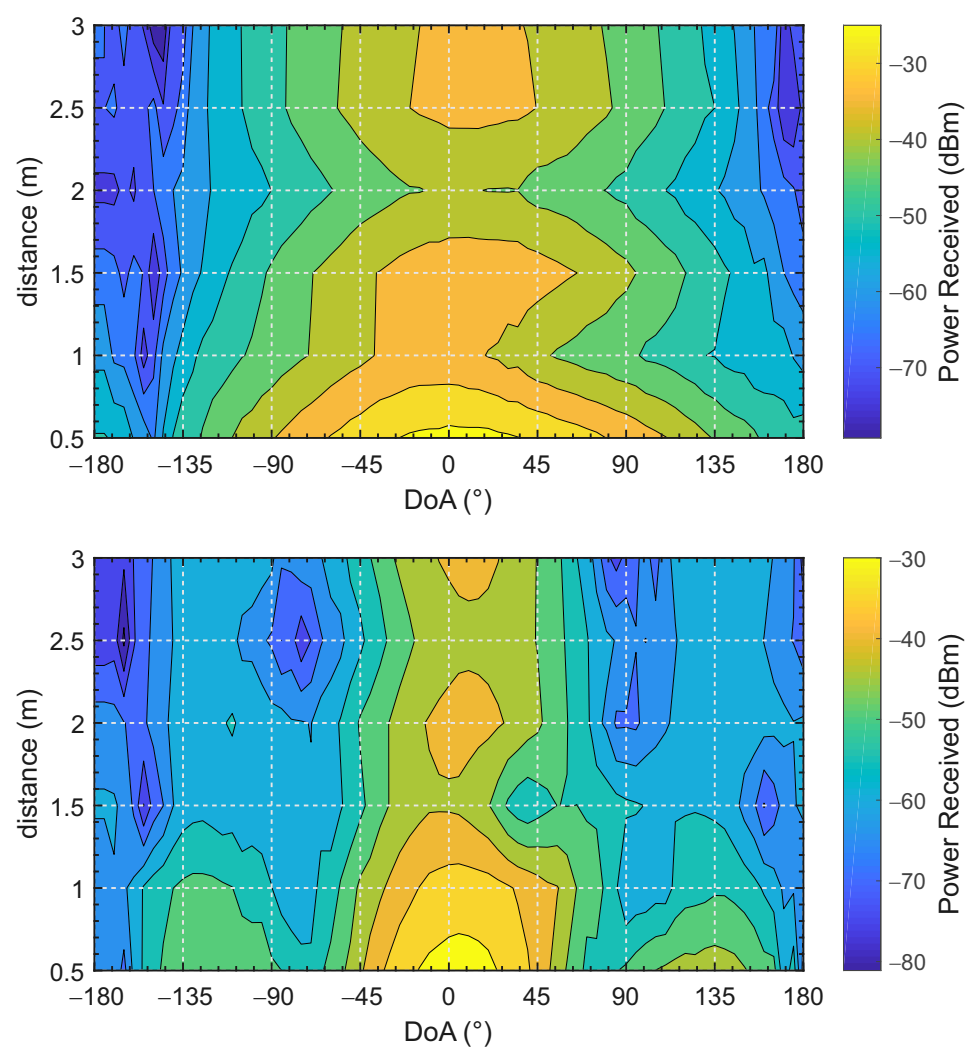

Figure 8. Power distribution relative to antenna \#1 in the case of a severe interference from the image antenna, at $2.45 \mathrm{GHz}$ (top) and $5.2 \mathrm{GHz}$ (bottom).

\section{Validation of the Simulation Environment}

The validation of the approach introduced in the previous paragraph is the goal of the present section. Virtual scenarios implemented by means of the aforementioned proposed techniques and subsequent investigations, performed to reach the highlighted objective, make use of the following parameters: the actual azimuthal DoA is set equal to $0^{\circ}$ while the distance $D$ ranges from $0.5 \mathrm{~m}$ to $3 \mathrm{~m}$. For each study case, the Image antenna (I) is kept $2 \mathrm{~m}$ away from the Target $(\mathrm{T})$, to explore a wide range of direction of interference signal. These parameters define a set of virtual scenarios compatible with the implementation of corresponding physical experiment.

The first validation step consisted of evaluating the simulated power distribution for one of the 8 antennas (the one pointing toward $\theta=0^{\circ}$ ) within the entire range of angles and distances considered and under the assumption that the Image antenna is inactive. The resulting power distribution is illustrated in Figure 7 where the shape of the pattern at the two frequencies is clearly highlighted. The latter maintains the shape of the power pattern along the distance of test. The second step was implemented by running the same simulation, but considering the Image antenna activated. In this case, the latter is fed with $\chi=1 \mathrm{~dB}$ with respect to the Target antenna, meaning a very severe multi-path. The effects of undesired signals recombination are clearly visible in Figure 8 that shows areas where the recombination is extremely destructive for both frequencies.

To further investigate the performance of the proposed simulation environment in terms of multi-path effects prediction, the calculated RSSI data from the two frequencies are synthesized, at three different distances $D_{1}=2 \mathrm{~m}, D_{2}=2.5 \mathrm{~m}$ and $D_{3}=3 \mathrm{~m}$. The data, virtually collected from both $\mathrm{T}$ and I antennas, are evaluated considering a variation 
on the entire phase range and an array of attenuation ranging from $\chi=0 \mathrm{~dB}$ to $\chi=10 \mathrm{~dB}$. This means that the actual T signal is affected by a decreasing level of aggressor signal.

The scanning of the whole possible phase range for DoA estimations is a consequence of the recombination effects running between $\mathrm{T}$ and I signals. The corresponding results for the mean error are shown in Figures 9-11, while Figures 12-14 refer to 90-th percentile errors.

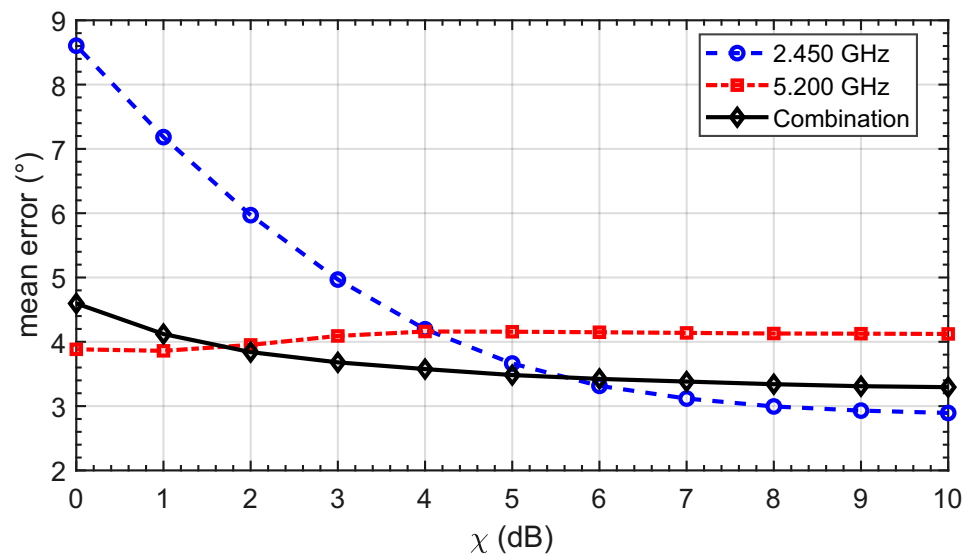

Figure 9. Mean DoA estimation error for $D=2 \mathrm{~m}$.

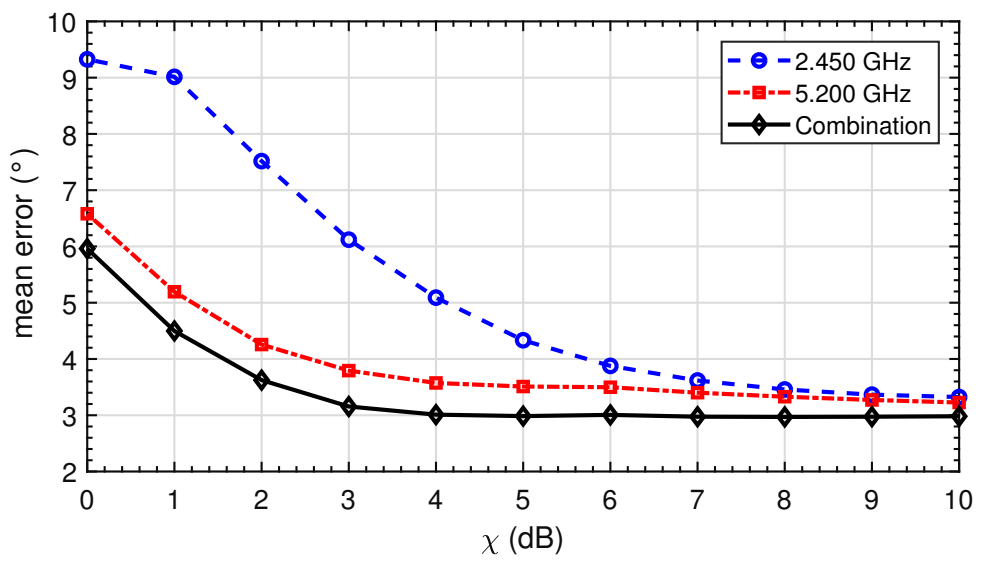

Figure 10. Mean DoA estimation error for $D=2.5 \mathrm{~m}$.

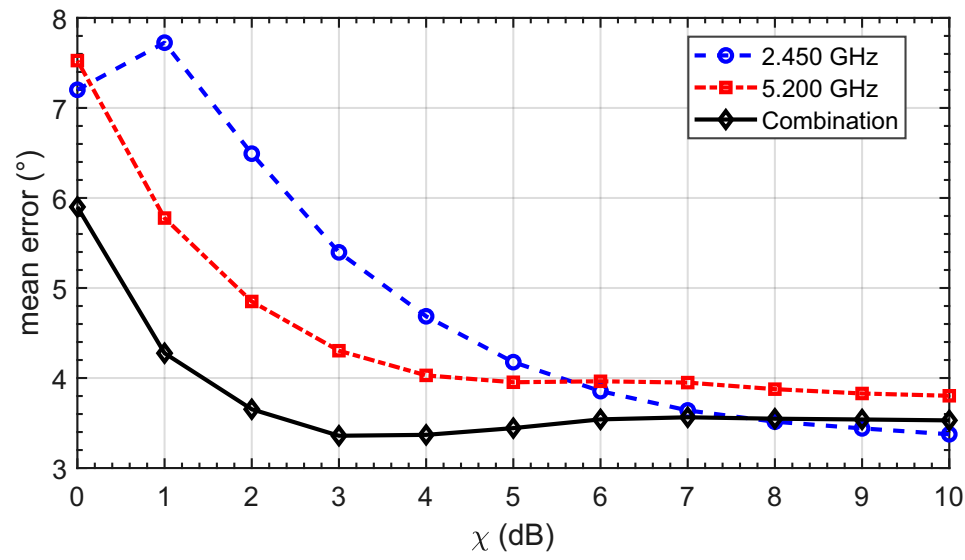

Figure 11. Mean DoA estimation error for $D=3 \mathrm{~m}$.

All figures above illustrate the results for both the two single frequencies, and the combination of the two datasets. These results, accordingly with the ones reported in [16] which were obtained from effective measurements, show how the estimation based on the combination of data extrapolated from the analysis of both two uncorrelated frequencies is 
almost always more accurate than the single one, or at least equivalent. This is true even if one of the two frequencies is severely impaired by multi-path.

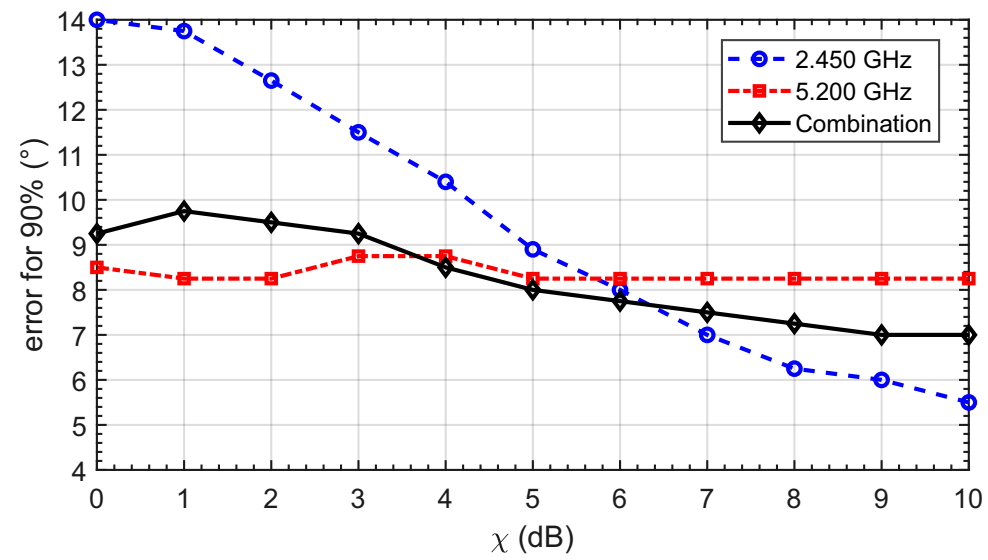

Figure 12. 90-th percentile DoA estimation error for $D=2 \mathrm{~m}$.

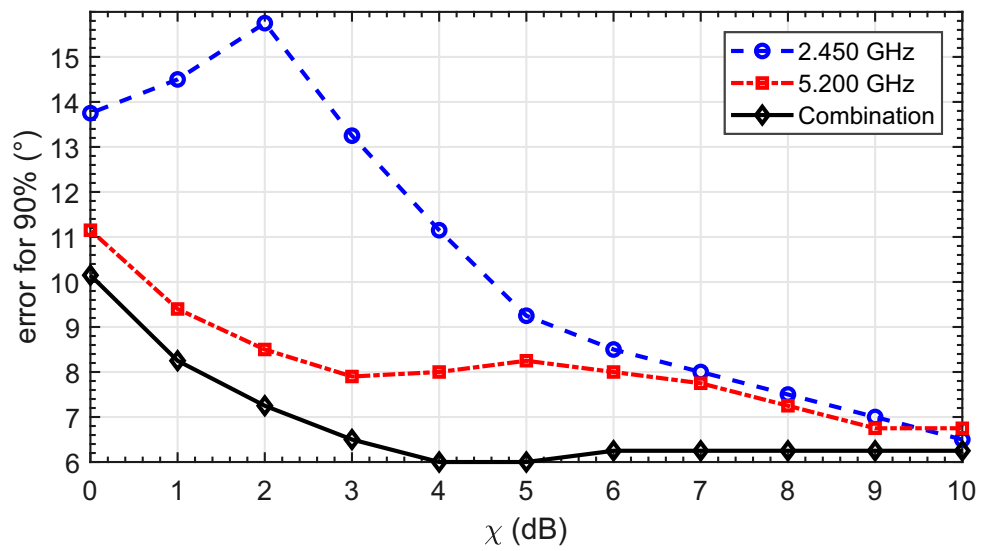

Figure 13. 90-th percentile DoA estimation error for $D=2.5 \mathrm{~m}$.

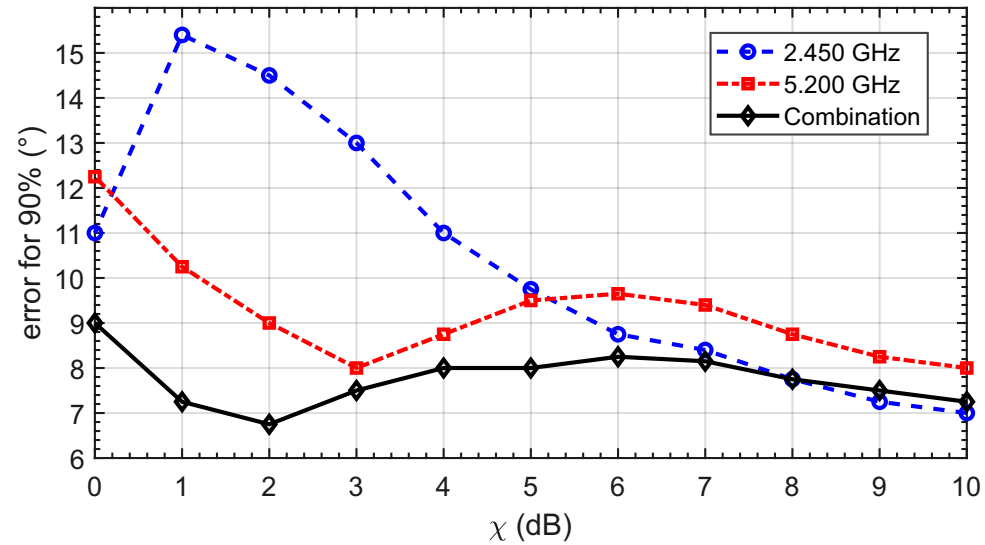

Figure 14. 90-th percentile DoA estimation error for $D=3 \mathrm{~m}$.

Given the fact that a reduced number of positive results can be fortuitous, the same analysis has been performed considering all five available distances for measurement results, cumulatively. The data obtained from this simulation are presented in Figures 15 and 16. These graphs have the same meaning of those already presented in previous figures. This comparison shows how it is not always the same frequency that performs worse than the other. In particular, the $2.45 \mathrm{GHz}$ signal has poorer performance 
up to around $\chi=5 \mathrm{~dB}$, then we have an inversion and is $5.2 \mathrm{GHz}$ that decays in quality. From the experiments we can infer that the wireless channel becomes much more sensitive to multi-path effects at lower frequency than to the higher one. This is not surprising because losses due to the pathloss at higher frequencies make the bounced signal weaker than the direct one. In addition, the antenna element adopted in the reference system exhibits a higher directivity at $5.2 \mathrm{GHz}$ as shown in Figure 3. All these contribute to reduce multi-path impairments at higher frequency, and thus avoid the peak reported in Figures 11 and 13-16. Consequently, the selection strategy between the two frequencies is carried out by the final identification procedure that weights for each set of simulated data the response at both the frequencies by their variance: this led to the extinction of the peak from the combined results. Anyway, the data combination enhances both mean and 90-th percentile error, as expected from measurements.

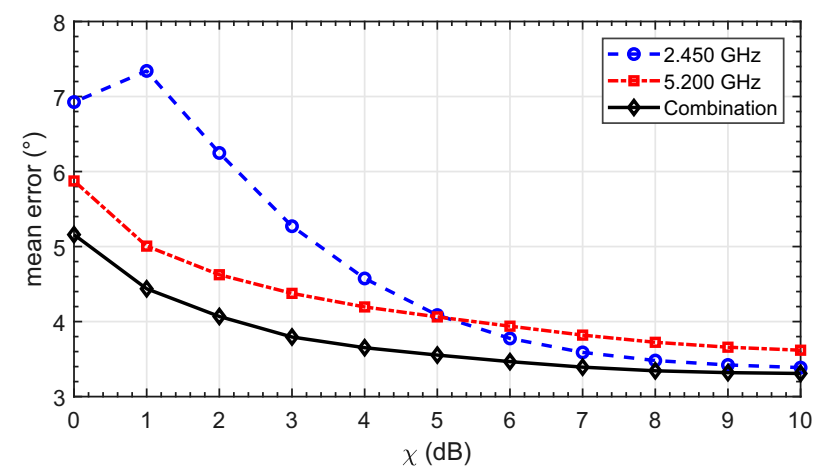

Figure 15. Mean DoA estimation error, synthetic graph for all $D$ values.

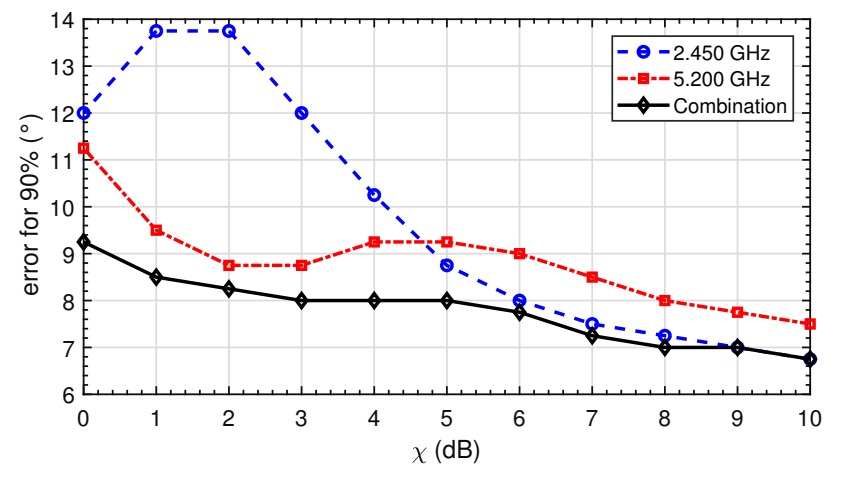

Figure 16. 90-th percentile DoA estimation error, synthetic graph for all $D$ values.

A further step has been carried out to fully validate the proposed method. The latter refers to a direct comparison between simulated and measured results. With reference to the simulated scenarios described at the beginning of the present section, three real scenarios were implemented for $D$ ranging from $2 \mathrm{~m}$ to $3 \mathrm{~m}$, in according to those described in [16]. An attenuation range from $\chi=0 \mathrm{~dB}$ to $\chi=10 \mathrm{~dB}$ has been used.

Figures 17-19 illustrate the comparison between the experimental results and the simulated ones for both Mean DoA estimation and 90-th percentile DoA estimation error, considering the combined use of the two analysis frequencies. The results depicted in Figure 17, and corresponding to $D=2 \mathrm{~m}$, show an optimum agreement between simulation and measurement in both Mean DoA estimation error and 90-th percentile estimation error. Maximum discrepancies of about $1.5^{\circ}$ and $2^{\circ}$ exist in Mean DoA and in 90-th percentile estimation error, respectively. Conversely, Figure 18 exhibits a difference of $4^{\circ}$ and $7^{\circ}$ respectively in Mean DoA error and 90-th percentile error, between simulation and measure at $\chi=0$ with $D=2.5 \mathrm{~m}$. Comparison results for $D=3 \mathrm{~m}$ are highlighted in Figure 19 , where at $\chi=0$ values of about $6.5^{\circ}$ and $4^{\circ}$ are achieved. 

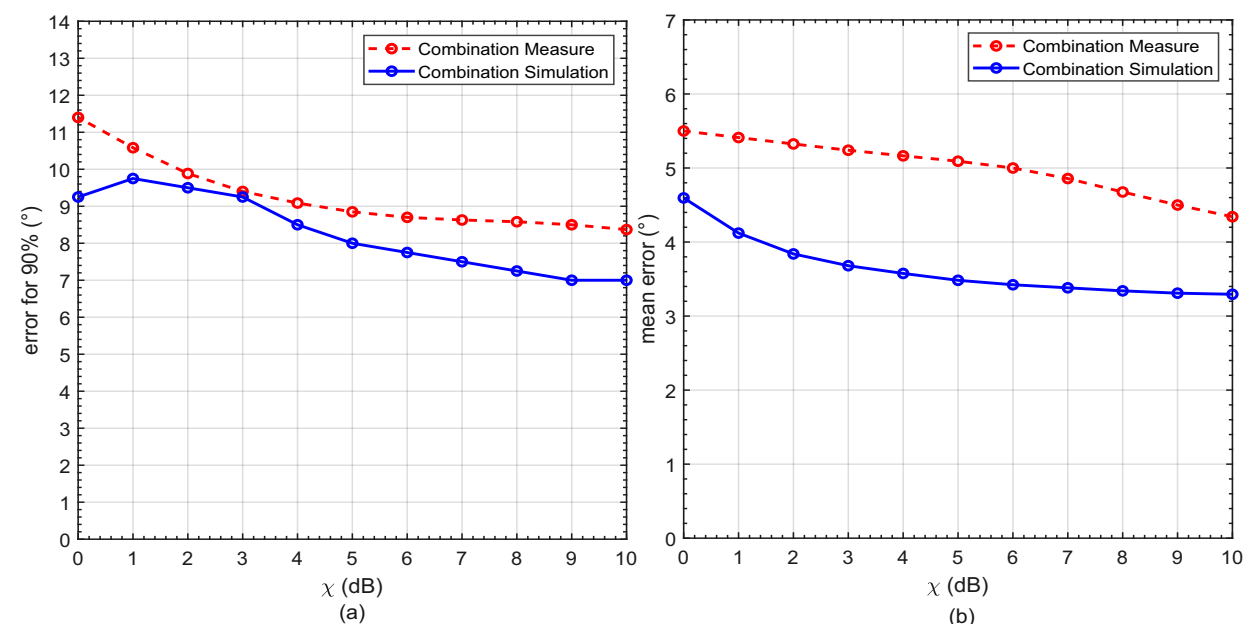

Figure 17. Comparison for $D=2 \mathrm{~m}$ between measurement and simulation environment of 90-th percentile combined DoA estimation error (a) and Mean combined DoA estimation error (b).

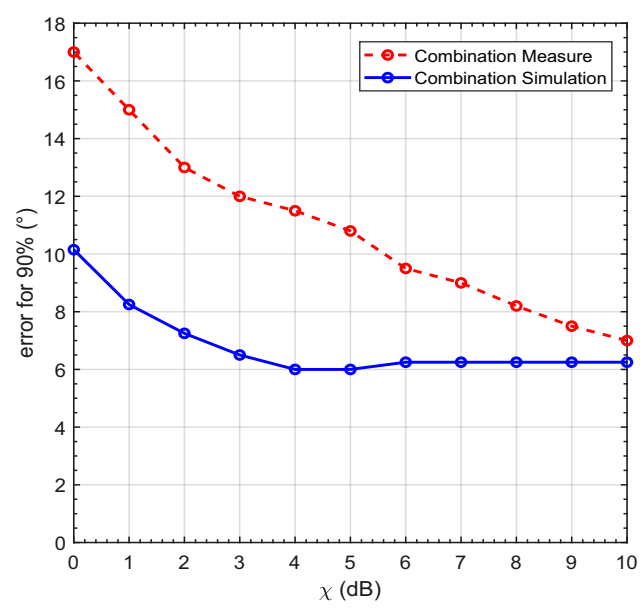

(a)

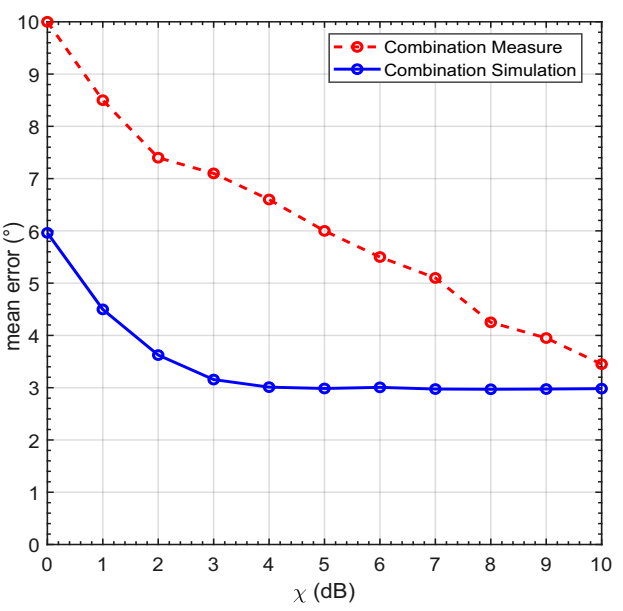

(b)

Figure 18. Comparison for $D=2.5 \mathrm{~m}$ between measurement and simulation environment of 90-th percentile combined DoA estimation error (a) and Mean combined DoA estimation error (b).

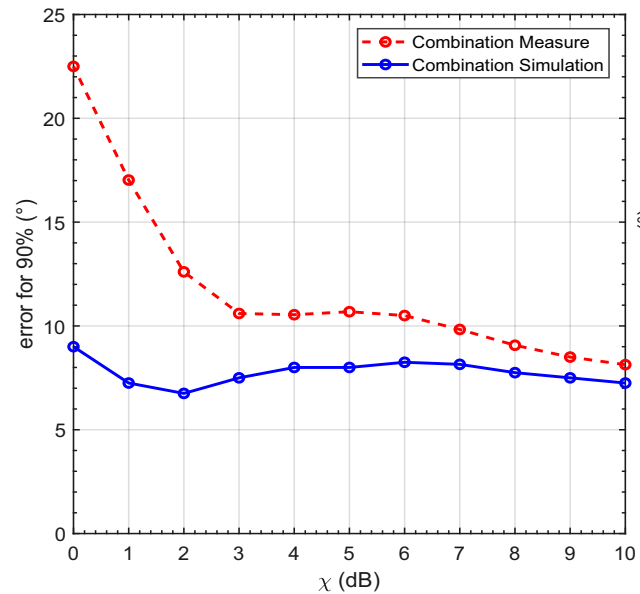

(a)

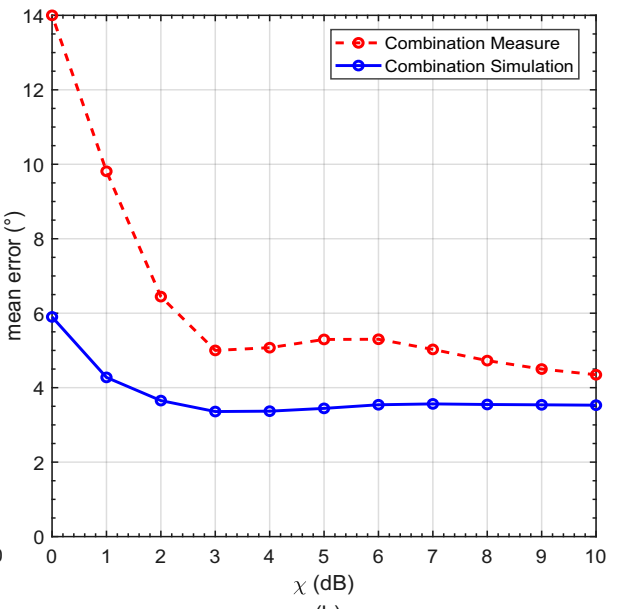

(b)

Figure 19. Comparison for $D=3 \mathrm{~m}$ between measurement and simulation environment of 90-th percentile combined DoA estimation error (a) and Mean combined DoA estimation error (b). 
The above results show how both errors decrease in the case of shorter distance, and even though the analysis method slightly underestimates the DoA, confirm the ability to predict correctly performance of the dual-band approach applied to real scenarios.

This important result represents a key objective in assessing the ability of this method to overcome the limitation involved in a real-world scenario to fully exploit the proposed new dual-band methodology for DoA-based localization philosophy.

\section{Conclusions}

This paper has described and validated a simulation environment designed to predict the performance of indoor localization systems based on dual-band DoA approach in a complex multi-path scenario. The proposed approach is implemented by means of a virtual environment defined through a set of full-wave simulations and controlled via an ideal circuital network. The latter, by connecting both the Target and his corresponding Image antenna, enables the full implementation of the two-ray reflection model.

As illustrated in Section 5, the validation process of the simulated results is reached by modeling the reference scenarios described in previous papers and exploiting the ability to predict the corresponding measurements, obtained experimentally.

This approach allows consideration of complex multi-path effects that are extremely difficult to model in real experimental scenario. Consequently, the use of the proposed simulation environment lets the designer to avoid a lengthy and difficult measurement campaign as well as long design step and makes it a predictive optimization tool for localization devices.

Future works could be done regarding the inclusion of a multi-path model, with a statistical meaning, as for example those generated by reverberation chambers. In addition, the development of a simulation environment for ultra-wideband DoA-based indoor positioning systems may be investigated.

Author Contributions: Conceptualization, A.C., G.C., S.M. and M.P.; methodology, S.M., M.P.; software, E.C. and M.P.; validation, A.C. and G.C.; formal analysis, S.M.; investigation, E.C., G.C., M.P.; resources, G.C.; data curation, S.M.; writing-original draft preparation, S.M.; writing-review and editing, E.C. and G.C.; visualization, A.C. and G.P.; supervision, A.C., G.C. and G.P.; project administration, G.P.; funding acquisition, G.C. All authors have read and agreed to the published version of the manuscript.

Funding: This research was funded by Regione Toscana POR CreO FESR 2014-2020-Action 1.1.5.a3 and resources of the Regione Toscana, Bando FAR-FAS 2014 under project "SUMA-Struttura Urbana Multifunzionale Attiva".

Data Availability Statement: The data presented in this study are available in the present article.

Conflicts of Interest: The authors declare no conflict of interest.

\section{References}

1. Guan, W.; Chen, S.; Wen, S.; Tan, Z.; Song, H.; Hou, W. High-Accuracy Robot Indoor Localization Scheme Based on Robot Operating System Using Visible Light Positioning. IEEE Photonics J. 2020, 12, 1-16. [CrossRef]

2. Khelifi, F.; Bradai, A.; Benslimane, A.; Rawat, P.; Atri, M. A Survey of Localization Systems in Internet of Things. Mob. Netw. Appl. 2019, 24, 761. [CrossRef]

3. El Boudani, B.; Kanaris, L.; Kokkinis, A.; Kyriacou, M.; Chrysoulas, C.; Stavrou, S.; Dagiuklas, T. Implementing Deep Learning Techniques in 5G IoT Networks for 3D Indoor Positioning: DELTA (DeEp Learning-Based Co-operaTive Architecture). Sensors 2020, 20, 5495. [CrossRef]

4. Guan, T.; Dong, W.; Koutsonikolas, D.; Challen, G.; Qiao, C. Robust, Cost-Effective and Scalable Localization in Large Indoor Areas. In Proceedings of the 2015 IEEE Global Communications Conference (GLOBECOM), San Diego, CA, USA, 6-10 December 2015; pp. 1-6. [CrossRef]

5. AlHajri, M.I.; Ali, N.T.; Shubair, R.M. Indoor Localization for IoT Using Adaptive Feature Selection: A Cascaded Machine Learning Approach. IEEE Antennas Wirel. Propag. Lett. 2019, 18, 2306-2310. [CrossRef]

6. Yan, J.; Zhao, L.; Tang, J.; Chen, Y.; Chen, R.; Chen, L. Hybrid Kernel Based Machine Learning Using Received Signal Strength Measurements for Indoor Localization. IEEE Trans. Veh. Technol. 2018, 67, 2824-2829. [CrossRef] 
7. Zhao, L.; Wang, H.; Wang, J.; Gao, H.; Liu, J. Robust Wi-Fi indoor localization with KPCA feature extraction of dual band signals. In Proceedings of the 2017 IEEE International Conference on Robotics and Biomimetics (ROBIO), Macau, China, 5-8 December 2017; pp. 908-913. [CrossRef]

8. Guan, T.; Dong, W.; Koutsonikolas, D.; Qiao, C. Fine-Grained Location Extraction and Prediction with Little Known Data. In Proceedings of the 2017 IEEE Wireless Communications and Networking Conference (WCNC), San Francisco, CA, USA, 19-22 March 2017; pp. 1-6. [CrossRef]

9. Maddio, S.; Passafiume, M.; Cidronali, A.; Manes, G. A scalable distributed positioning system augmenting WiFi technology. In Proceedings of the International Conference on Indoor Positioning and Indoor Navigation, Montbeliard, France, 28-31 October 2013; pp. 1-10. [CrossRef]

10. Cidronali, A.; Maddio, S.; Giorgetti, G.; Magrini, I.; Gupta, S.K.S.; Manes, G. A 2.45 GHz smart antenna for location-aware single-anchor indoor applications. In Proceedings of the 2009 IEEE MTT-S International Microwave Symposium Digest, Boston, MA, USA, 7-12 June 2009; pp. 1553-1556. [CrossRef]

11. Tran, H.Q.; Ha, C. Fingerprint-Based Indoor Positioning System Using Visible Light Communication-A Novel Method for Multipath Reflections. Electronics 2019, 8, 63. [CrossRef]

12. Nessa, A.; Adhikari, B.; Hussain, F.; Fernando, X.N. A Survey of Machine Learning for Indoor Positioning. IEEE Access 2020, 8, 214945-214965. [CrossRef]

13. Wielandt, S.; Strycker, L.D. Indoor Multipath Assisted Angle of Arrival Localization. Sensors 2017, 17, 2522. [CrossRef]

14. Li, Z.; Tian, Z.; Wang, Z.; Zhang, Z. Multipath-Assisted Indoor Localization Using a Single Receiver. IEEE Sens. J. 2021, 21, 692-705. [CrossRef]

15. Tao, C.; Zhou, B. Indoor Localization with Smart Antenna System: Multipath Mitigation with MIMO Beamforming Scheme. In Proceedings of the 2017 IEEE 14th International Conference on Mobile Ad Hoc and Sensor Systems (MASS), Orlando, FL, USA, 22-25 October 2017; pp. 303-307. [CrossRef]

16. Maddio, S.; Cidronali, A.; Passafiume, M.; Collodi, G.; Lucarelli, M.; Maurri, S. Multipath Robust Azimuthal Direction of Arrival Estimation in Dual-Band 2.45-5.2 GHz Networks. IEEE Trans. Microw. Theory Tech. 2017, 65, 4438-4449. [CrossRef]

17. Hood, B.N.; Barooah, P. Estimating DoA From Radio-Frequency RSSI Measurements Using an Actuated Reflector. IEEE Sens. J. 2011, 11, 413-417. [CrossRef]

18. Passafiume, M.; Maddio, S.; Lucarelli, M.; Cidronali, A. An enhanced triangulation algorithm for a distributed RSSI-DoA positioning system. In Proceedings of the 2016 European Radar Conference (EuRAD), London, UK, 5-7 October 2016; pp. 185188.

19. Zafari, F.; Gkelias, A.; Leung, K.K. A survey of indoor localization systems and technologies. IEEE Commun. Surv. Tuts. 2019, 21, 2568-2599. [CrossRef]

20. Liu, H.; Darabi, H.; Banerjee, P.; Liu, J. Survey of wireless indoor positioning techniques and systems. IEEE Trans. Syst. Man Cybern. C Appl. Rev. 2007, 37, 1067-1080. [CrossRef]

21. Chen, L.; Pei, L.; Kuusniemi, H.; Chen, Y.; Kröger, T.; Chen, R. Bayesian fusion for indoor positioning using Bluetooth fingerprints. Wireless Pers. Commun. 2013, 70, 1735-1745. [CrossRef]

22. Dardari, D.; Conti, A.; Ferner, U.; Giorgetti, A.; Win, M.Z. Ranging with ultrawide bandwidth signals in multipath environments. Proc. IEEE 2009, 97, 404-426. [CrossRef]

23. Kalis, A.; Antonakopoulos, T. Direction finding in IEEE802.11 wireless networks. IEEE Trans. Instrum. Meas. 2002, 51, 940-948. [CrossRef]

24. Nagah Amr, M.; ELAttar, H.M.; Abd El Azeem, M.H.; El Badawy, H. An Enhanced Indoor Positioning Technique Based on a Novel Received Signal Strength Indicator Distance Prediction and Correction Model. Sensors 2021, 21, 719. [CrossRef]

25. Fang, S.; Lin, T. A dynamic system approach for radio location fingerprinting in wireless local area networks. IEEE Trans. Commun. 2010, 58, 1020-1025. [CrossRef]

26. Fang, S.; Lin, T. Indoor Location System Based on Discriminant-Adaptive Neural Network in IEEE 802.11 Environments. IEEE Trans. Neural Netw. 2008, 19, 1973-1978. [CrossRef]

27. Denkovski, D.; Angjelicinoski, M.; Atanasovski, V.; Gavrilovska, L. Practical assessment of RSS-based localization in indoor environments. In Proceedings of the MILCOM 2012-2012 IEEE Military Communications Conference, Orlando, FL, USA, 29 October-1 November 2012; pp. 1-6. [CrossRef]

28. Lee, J.; Jo, S. Improvement of exhaustive search based maximum-likelihood DOA estimation. In Proceedings of the IEEE 2010 National Aerospace Electronics Conference, Fairborn, OH, USA, 14-16 July 2010; pp. 246-249. [CrossRef]

29. Maddio, S.; Passafiume, M.; Cidronali, A.; Manes, G. Impact of the dihedral angle of switched beam antennas in indoor positioning based on RSSI. In Proceedings of the 2014 11th European Radar Conference, Rome, Italy, 8-10 October 2014; pp. 317-320.

30. Nehorai, A.; Starer, D.; Stoica, P. Direction of Arrival estimation in applications wth multipath and few snapshots. Circuits Syst. Signal Process. 1991, 10, 327-342. [CrossRef]

31. Cidronali, A.; Collodi, G.; Maddio, S.; Passafiume, M.; Pelosi, G.; Selleri, S. Improving Phaseless Direction of Arrival (DoA) Estimation in Multipath-Impaired Scenarios by Exploiting Dual-Band Operations. In Proceedings of the 2016 Conf. Int. Microwave Symposium (IMS), San Francisco, CA, USA, 22-27 May 2016; pp. 1-4.

32. Cidronali, A.; Collodi, G.; Maddio, S.; Passafiume, M.; Pelosi, G. 2-D DoA Anchor Suitable for Indoor Positioning Systems Based on Space and Frequency Diversity for Legacy WLAN. IEEE Microw. Wirel. Components Lett. 2018, 28, 627-629. [CrossRef] 
33. Loyka, S.; Kouki, A. Using two ray multipath model for microwave link budget analysis. IEEE Antennas Propag. Mag. 2001, 43, 31-36. [CrossRef]

34. Szumny, R.; Kurek, K.; Modelski, J. Attenuation of multipath components using directional antennas and circular polarization for indoor wireless positioning systems. In Proceedings of the 2007 European Microwave Conference, Munich, Germany, 8-12 October 2007; pp. 1680-1683. [CrossRef]

35. Thomas, T.A.; Vook, F.W.; Sun, S. Investigation into the effects of polarization in the indoor mmWave environment. In Proceedings of the 2015 IEEE International Conference on Communications (ICC), London, UK, 8-12 June 2015; pp. 1386-1391. [CrossRef]

36. Kanaris, L.; Kokkinis, A.; Raspopoulos, M.; Liotta, A.; Stavrou, S. Improving RSS fingerprint-based localization using directional antennas. In Proceedings of the 8th European Conference on Antennas and Propagation (EuCAP 2014), Hague, The Netherlands, 6-11 April 2014; pp. 1593-1597. [CrossRef]

37. Maddio, S.; Cidronali, A.; Magrini, I.; Manes, G. A design method for single-feed wideband microstrip patch antenna for switchable circular polarization. In Proceedings of the 2007 European Microwave Conference, Munich, Germany, 8-10 October 2007; pp. 262-265. [CrossRef]

38. Maddio, S.; Passafiume, M.; Cidronali, A.; Manes, G. A Distributed Positioning System Based on a Predictive Fingerprinting Method Enabling Sub-Metric Precision in IEEE 802.11 Networks. IEEE Trans. Microw. Theory Tech. 2015, 63, 4567-4580. [CrossRef]

39. Passafiume, M.; Maddio, S.; Cidronali, A. An improved approach for RSSI-based only calibration-free real-time indoor localization on IEEE 802.11 and 802.15. 4 wireless networks. Sensors 2017, 17, 717. [CrossRef] [PubMed] 